\title{
Effect of additives on suspension pipe flow
}

\author{
UW Graham CSIRO Mineral Resources, Australia
}

G Short CSIRO Mineral Resources, Australia

A Chryss CSIRO Mineral Resources, Australia

K Constanti-Carey CSIRO Mineral Resources, Australia

J Wu CSIRO Mineral Resources, Australia

\begin{abstract}
Suspension pipe flow is an important part of mineral operations, which can involve concentrate transport, intra-plant transport, tailings disposal and backfill. Significant costs in these operations include energy, capital and maintenance (the latter associated with the repair of erosion damage). Full understanding of the flow regime is required to optimise the design of these systems for the desired operating condition. An additional option available to the pipe designer or operator is that additives such as magnesium aluminosilicate (MAS) can be used to change the flow regime of the pipe system to the advantage of the operation. A recent program has investigated some of these aspects of pipe flow experimentally.
\end{abstract}

It was found that:

- Regardless of the additives to the test slurry, stratified flow was observed in the pipe.

- The combination of highly purified MAS and salt as additives led to a reduced pressure gradient in the pipe. This combination also resulted in an easier pipe restart from shutdown. The effect is most likely due to the modification of the bed of solids and its frictional behaviour with the changed rheology, particularly in turbulent flows. The minimum conveying velocity was also reduced.

- It was possible to restart without problems after two days for the $0.75 \%$ salt and $0.1 \%$ MAS case. This would have not been attempted with the lower-viscosity control suspensions, due to the risk of blockage.

- Samples taken from the pipe tests showed markedly different behaviour for the MAS plus salt case compared with the other samples, with little segregation of the different size fractions.

- Calculations indicate that increasing the viscosity of homogeneous suspensions or carrier fluids in heterogeneous suspensions can be beneficial for pipe flow in the turbulent regime.

Future research options could include examining the effect of additives on different clay-based model suspensions and industrial samples.

Keywords: non-Newtonian, pipe, additives, rheology, magnesium aluminosilicate

\section{Introduction}

Suspension pipe flow is an important part of mineral operations, which can involve concentrate transport, intra-plant transport, tailings disposal and backfill. Significant costs in these operations can include energy, capital and maintenance, with the latter typically associated with the repair of erosion damage.

Full understanding of the flow regime is required to optimise the design of these systems for the desired operating condition. An additional option available to the pipe designer or operator is that additives can be used to change the flow regime of the pipe system to the advantage of the operation. For example, raising 
the carrier fluid viscosity can make the pipe more resistant to blockage events and make it easier to restart the system from stoppages.

A particular case of this is when the pipe flow is at the lower carrier fluid viscosity range, which can lead to issues where the flow develops a definite minimum conveying velocity and consequent risk of blockage unless the flow velocity exceeds the specified minimum at all times. At the 2014 19th International Conference on Hydrotransport (Sanders \& Sumner 2014) there was an entire session on the topic of minimum (or stationary deposit) velocity, thus demonstrating the importance of this concept to the pipe flow community. The literature contains many methods to calculate the minimum conveying velocity, as outlined by Thomas (2014), although there is no generally accepted method suitable for all slurry types, especially when non-Newtonian effects are present.

The present work examines the effect of additives on a suspension with a large particle size range, from clay sized particles up to $7 \mathrm{~mm}$ aggregate particles, mimicking a backfill material, in this case at the low-viscosity end of the spectrum. These suspensions can be approximated as a rheologically active fraction and a coarse fraction. Modification of the characteristics of the rheologically active fraction can have an effect on the deposition velocity and pressure gradient behaviour in pipe flow, and as such may lead to operational convenience if minimum conveying velocities can be reduced. Rheology and pipe flow tests were conducted to explore this behaviour. The effects of salt $(\mathrm{NaCl})$ and a highly purified magnesium aluminosilicate-based additive (referred to as 'MAS') on the behaviour of the suspension were investigated.

The specific research activities included:

- Development of a model suspension mimicking backfill material with readily available commercial clays, sands and gravels. This is considered to model a rheologically dominated carrier fluid together with a coarse solids fraction, which makes up the complete suspension. Particle size distributions were matched closely to typical industrial conditions.

- Rheological and pipe loop tests of this suspension 'as is' and with varying quantities of a MAS-based additive, as well as varying ionic strength with the addition of sodium chloride. The ionic strength of the process water is a controlling factor on the performance of the MAS.

- Measurements were made of the pipe flow conveying characteristics, with electrical resistance tomography (ERT) used to interrogate the suspension state of the coarse solids fraction.

\section{Experimental facility and materials}

The tilting pipe test facility at the CSIRO Clayton laboratories was used for the pipeline tests, although in horizontal mode only. The rig consists of a loop of ND100 (96 mm ID) PVC pipe measuring $20 \mathrm{~m}$ out and $20 \mathrm{~m}$ return. A 3,000 L agitated tank is used to supply the rig via a Warman 6/4 AH centrifugal pump. A magnetic flow meter is used to monitor the flow rate in the rig and pressure measurements are made using Rosemount ${ }^{\mathrm{TM}} 3051 \mathrm{CD}$ differential pressure transducers. A personal computer (PC), equipped with National Instruments 6008 and 6009 USB data acquisition hardware, is used for data logging and LabVIEW software to provide the necessary SCADA system. Apart from the conventional instrumentation, an ERT spool, a clear section of pipe and a sample station allowing samples to be taken from the top and bottom of the pipe, were inserted into the return length near the end of the line. A schematic diagram of the rig is shown in Figure 1. Samples for rheological analysis were taken from the top of the pipe at low velocity to minimise the amount of coarse solids in the sample. 


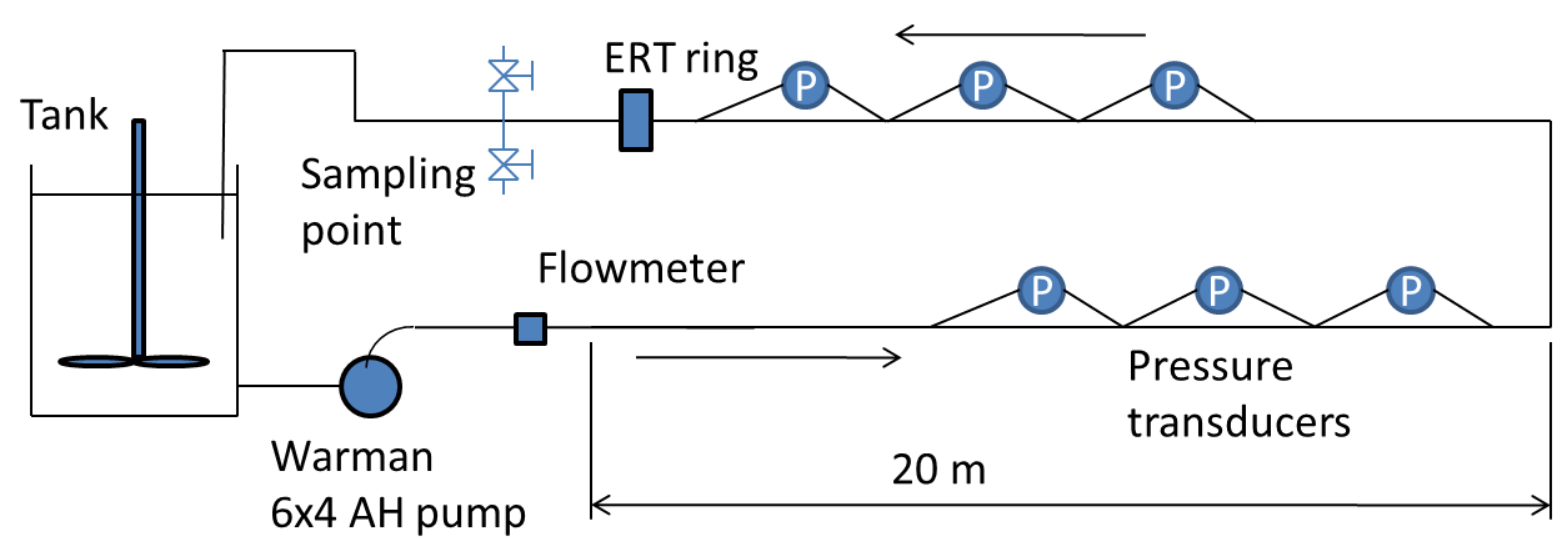

Not to scale

Figure 1 Schematic diagram of pipe rig

ERT was used to obtain qualitative images of the cross-section of the pipe flow. This technique provides an image of the electrical conductivity distribution in the pipe section. Given differing conductivities between the carrier fluid and the coarse solids phase, it is possible to determine whether stratification is occurring in the pipe flow.

The ERT system used was an ITS p2000, supplied by Industrial Tomography Systems plc (www.itoms.com). It is controlled by a PC. The technique requires that the pipe's cross-section be interrogated by injecting high-frequency current at various locations around the pipe's periphery and simultaneously measuring the electrical potential at other locations around the periphery. The conductivity map is then obtained from tomographic reconstruction of this data. A set of 16 electrodes plus one ground electrode are required to be in contact with the suspension in the pipe to do this. Such electrodes have been built into a weatherproof PVC enclosure which fits between two standard $100 \mathrm{~mm}$ pipe flanges on the pipe rig.

A reference dataset is normally required when using ERT, which is then used in conjunction with subsequent datasets. This is typically done by using a pure carrier fluid (e.g. water) in situ in the pipeline. A homogenous mixture of the synthetic tailings (discussed later in this paper) was placed in a short length of pipe with an ERT ring and used as the reference dataset. This qualitative method then allows the relative degree of stratification during the various pipe tests to be observed.

Rheological characterisation of the slurries was conducted using a Couette type (concentric cylinder) rheometer and a rotating vane test. Corrections for non-uniform shear rate in the Couette measuring gap were made, with the correct shear rate (allowing for the non-Newtonian fluid effect) obtained by using an integration approach for the Couette inverse problem. This method is generally more successful than a differential approach due to the inevitable noise present in real data. The inner and outer surfaces of the Couette geometry were profiled to minimise slip artefacts. The Couette tests were performed in a HAAKE ${ }^{\mathrm{TM}}$ RheoStress $^{\mathrm{TM}} 1$ Rheometer, with vane tests performed in a Bohlin CVO 50 controlled stress rheometer.

The fluid and coarse solids that comprise the backfill analogue were selected to best reflect the rheology, response to MAS and particle size distribution (PSD) of a cemented tailings sample. Bentonite was used as the rheologically active fraction, and the PSDs are shown in Figure 2 for the complete model suspension mix (including the silica and sand) and the tailings. It is seen they match closely. The model suspension components are shown in Table 1. 
Table 1 Nominal as loaded composition of test slurry into pipe rig

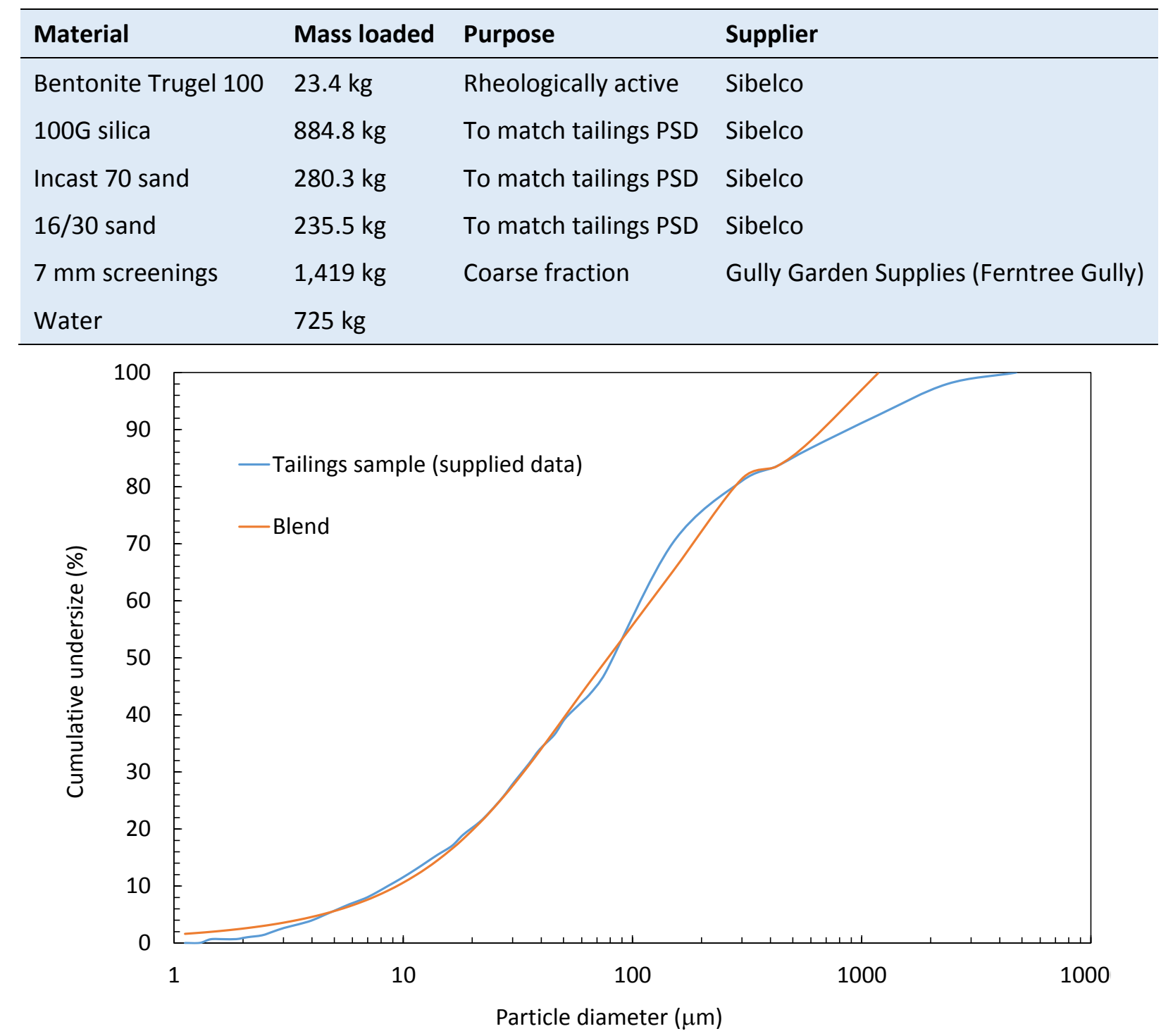

Figure 2 Particle size distribution of tailings sample compared with model suspension (compiled from supplier data)

\section{Results and discussion}

There were three batches of tests in the overall campaign, with a selection of results from these presented in this paper. The control suspension was the same for each test.

The 'as-loaded' composition of the suspension is given in Table 1. The nominal solids concentration is $80 \%$ by weight, though this does not take into account any residual water which may have been in the pipe rig or with the screenings.

Flow curves for a set of pipe tests are given in Figure 3. It is seen that the addition of the salt and MAS led to reduced pressure gradient as the additive levels were increased. The minimum conveying velocity of the control was estimated to be between $0.5-0.7 \mathrm{~m} \mathrm{~s}^{-1}$. As the additives were increased, it was possible to operate at a lower velocity $\left(0.3-0.4 \mathrm{~m} \mathrm{~s}^{-1}\right)$.

The pump power as read from the variable frequency drive is shown in Figure 4. It can be seen that the pump power is highest for the control and lowest for the control $+0.75 \%$ salt $+0.05 \%$ MAS. It is also of interest to note that the difference between the control and the control $+0.75 \%$ salt $+0.05 \%$ MAS case is largest at the 
highest velocity. This suggests that the effect of the carrier fluid rheology as modified by the salt and MAS is most apparent in the turbulent flow regime. This aspect is discussed further later in the paper.

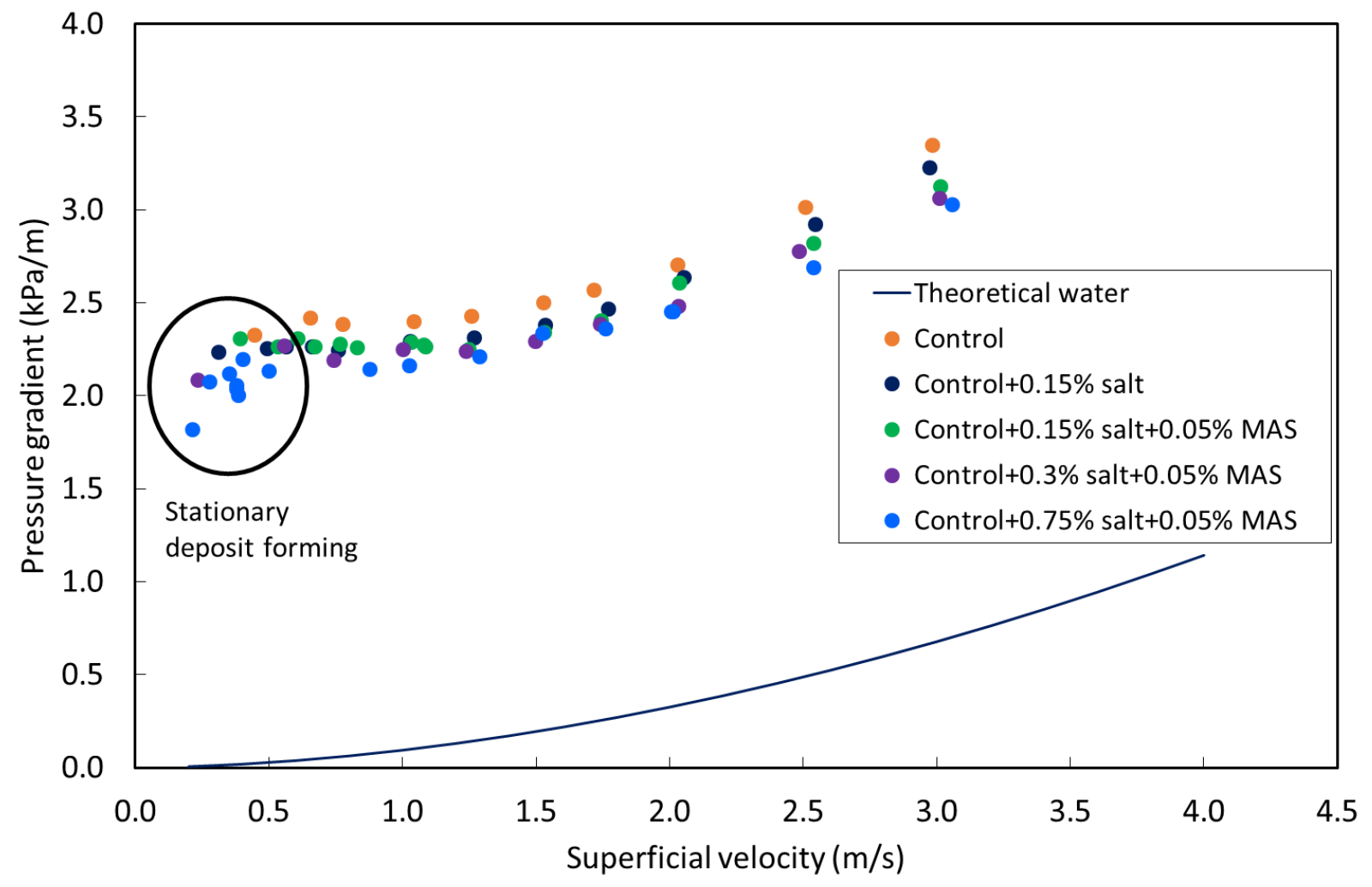

Figure 3 Flow curves for batch two test

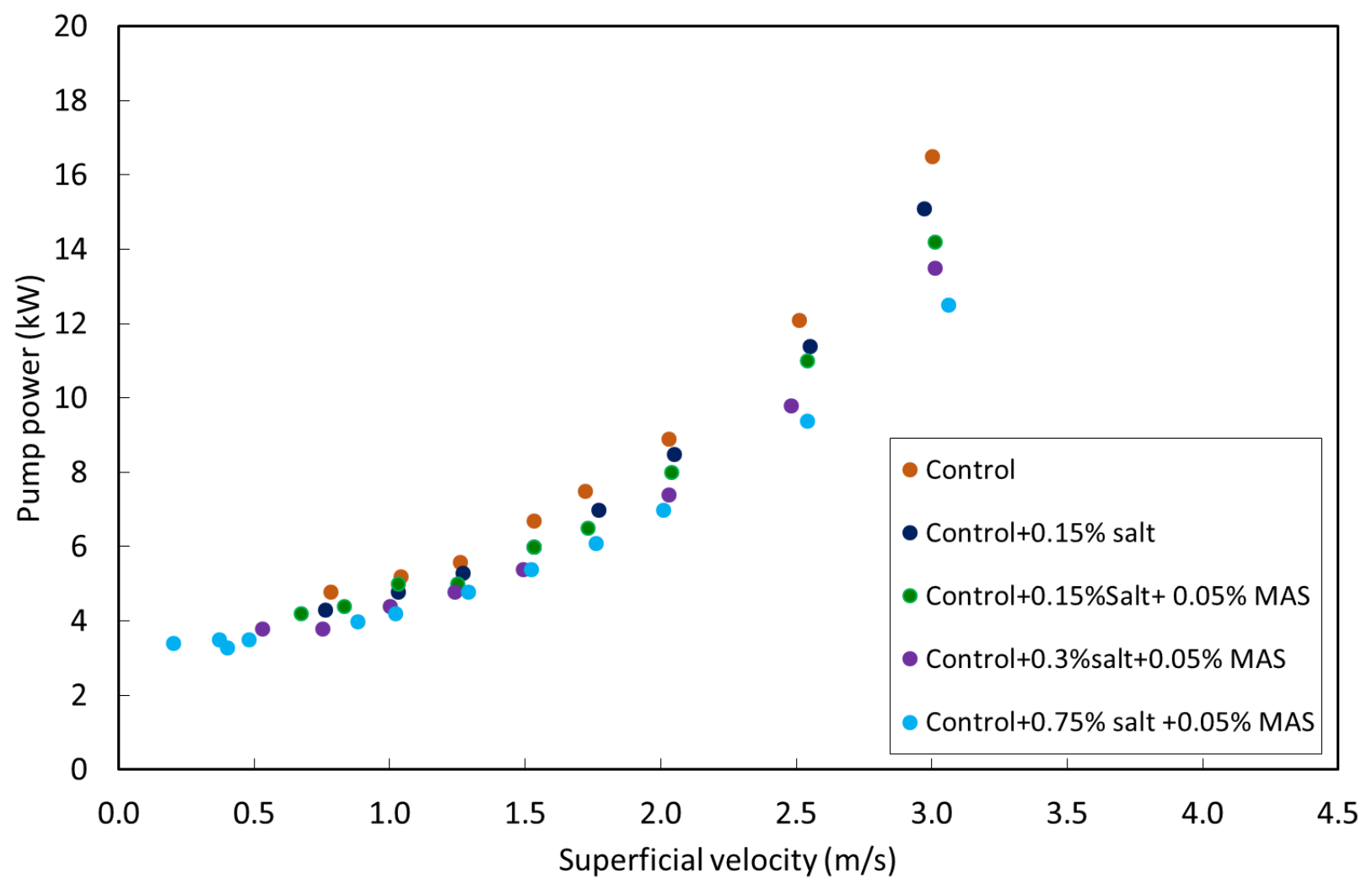

Figure 4 Pump power for batch two test 
The corresponding carrier fluid rheology for these tests is shown in Figure 5 where it can be seen that the combination of salt and MAS led to increased yield stresses and viscosity over a range of shear rates.

A sample set of ERT images is shown in Figure 6 for the control and Figure 7 for the case of $0.75 \%$ salt and $0.05 \%$ MAS. The images display stratification, increasing as the velocity decreases.

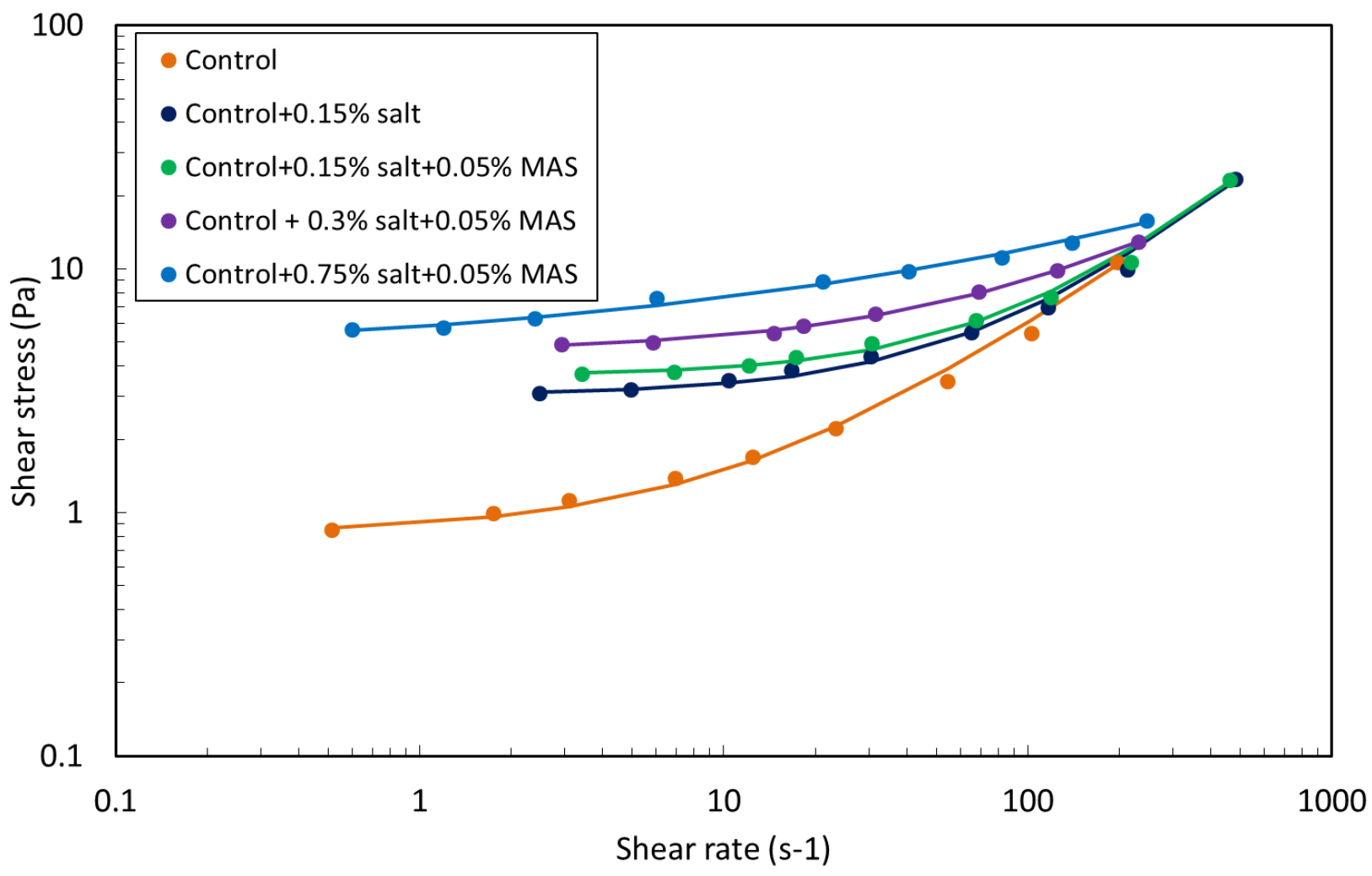

Figure 5 Rheology data from pipe tests samples for batch two test
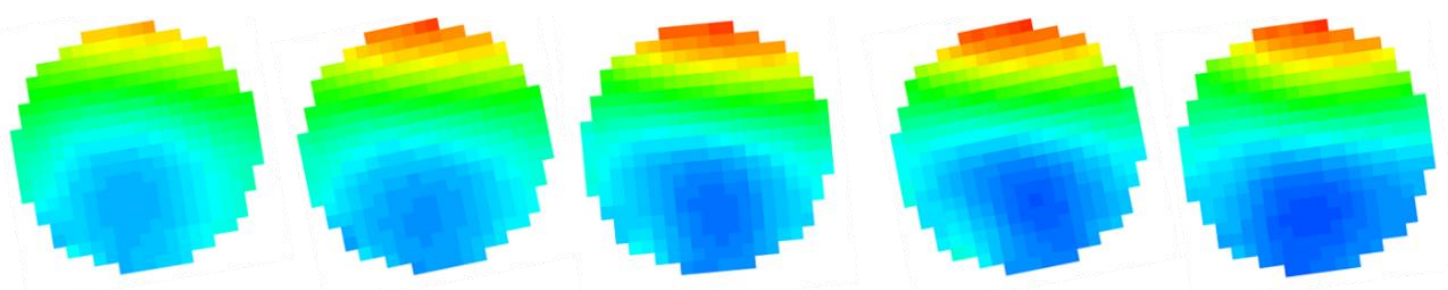

$2.5 \mathrm{~m} / \mathrm{s}$

$2.0 \mathrm{~m} / \mathrm{s}$

$1.7 \mathrm{~m} / \mathrm{s}$

$1.5 \mathrm{~m} / \mathrm{s}$

$1.0 \mathrm{~m} / \mathrm{s}$

Solids

Carrier

Figure 6 Electrical resistance tomography images from control for batch two test

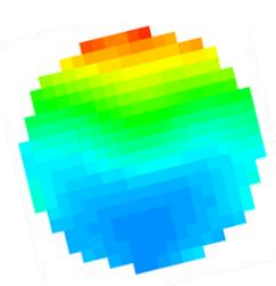

$2.5 \mathrm{~m} / \mathrm{s}$

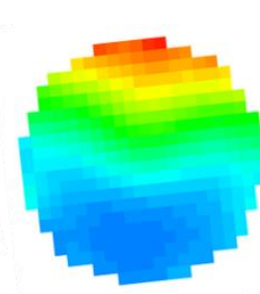

$2.0 \mathrm{~m} / \mathrm{s}$

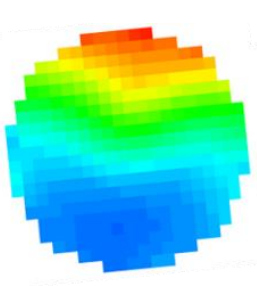

$1.76 \mathrm{~m} / \mathrm{s}$

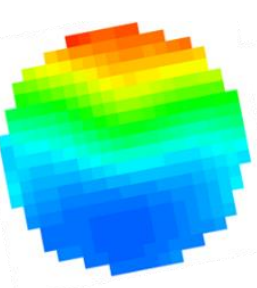

$1.5 \mathrm{~m} / \mathrm{s}$

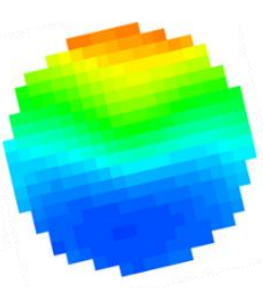

$1 \mathrm{~m} / \mathrm{s}$

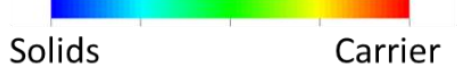

Figure 7 Electrical resistance tomography images from case with $0.75 \%$ salt $+0.05 \%$ MAS for batch two test 
The restart behaviour of the suspensions from a subsequent test is shown in Figures 8 and 9. All suspensions were restarted with the same setting on the variable-frequency drive (VFD) $(20 \mathrm{~Hz})$ after the same amount of stationary time with the exception of the two-day restart. The pressure signals in Figure 8 and the flow meter signals in Figure 9 show that the control suspension was more difficult to restart than any of the other suspensions and the VFD frequency had to be increased from $20 \mathrm{~Hz}$ to obtain sustained flow for the control suspension, as seen at the 30 second mark for this case. It was also possible to restart the system (including the mixing tank) after two days without any difficulty for the case with $0.75 \%$ salt and $0.1 \%$ MAS. The authors would not have attempted this with the lower viscosity control suspension, due to the risk of blockage.

It is expected that the detailed behaviour of a suspension together with additives will depend on the character of the suspension, such as ionic strength, $\mathrm{pH}$ level and species present and will be difficult to predict outside of pipe and/or rheology testing.

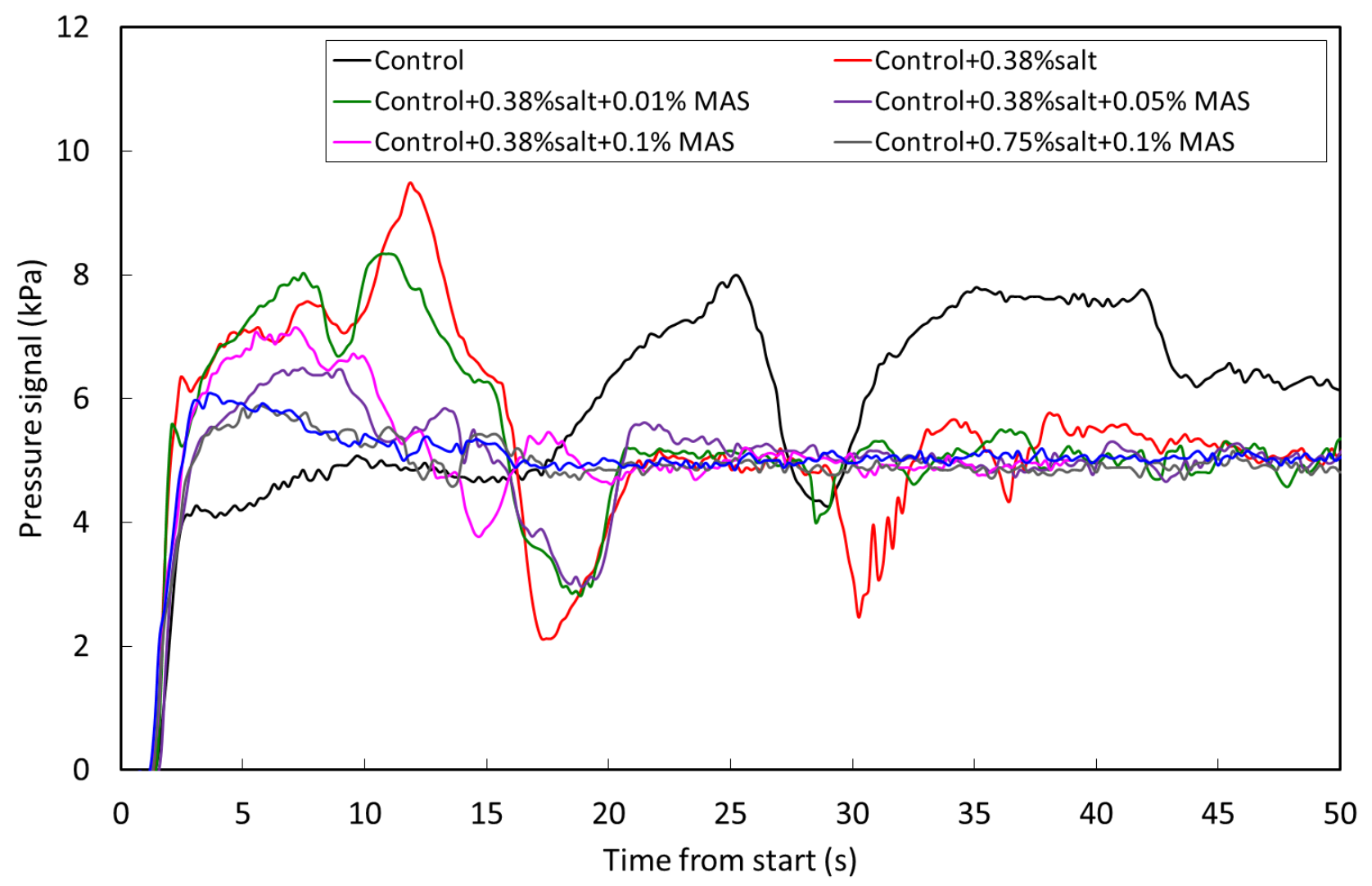

Figure 8 Pressure signals from transducer three during restart for batch three test 


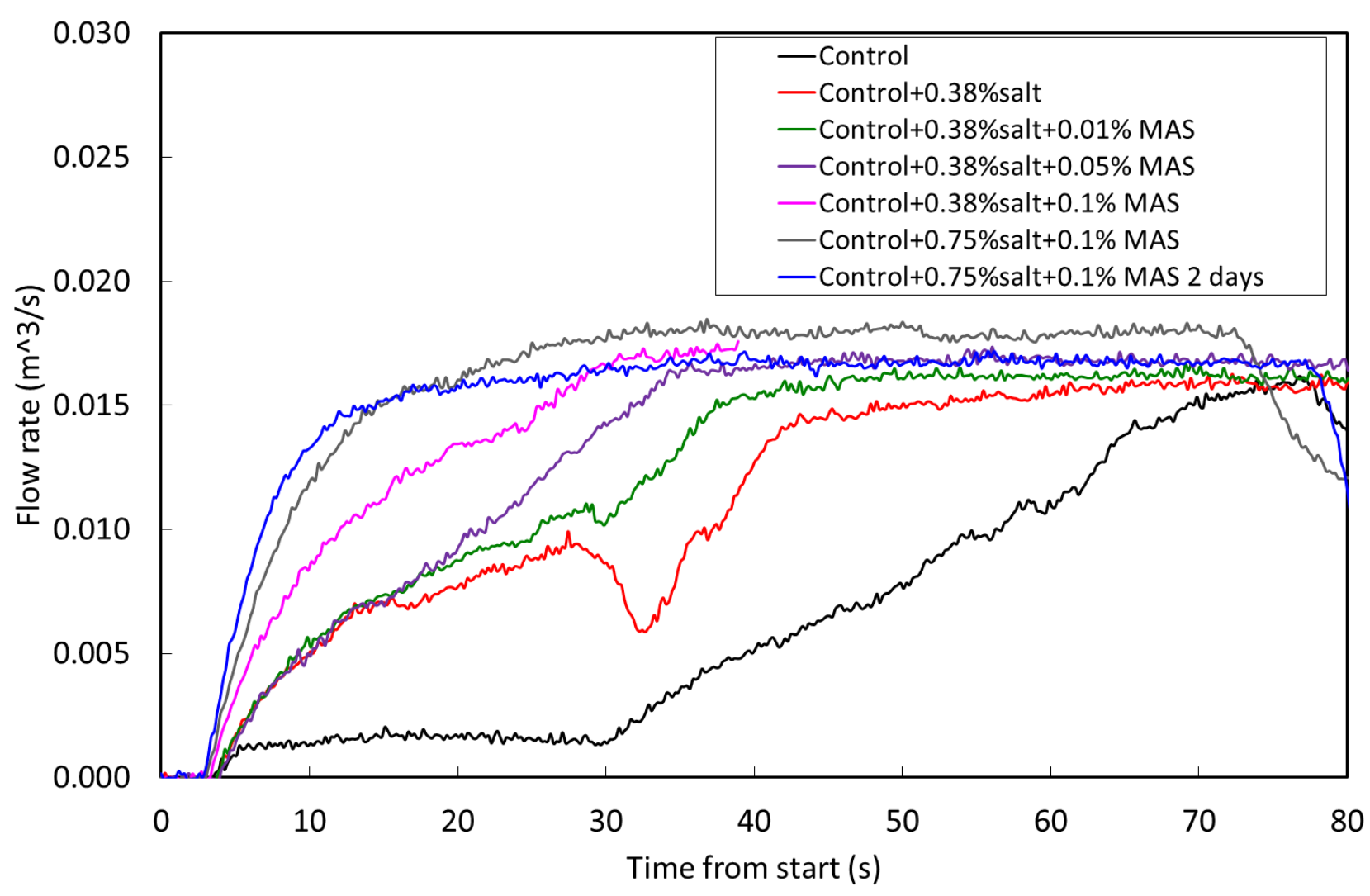

Figure 9 Flow rate signals during restart for batch three test

Table 2 shows calculations of the pressure gradient reduction for the first batch of tests, comparing the control with a case with $0.05 \%$ MAS $+0.015 \%$ salt. It should be noted that the results are from curve fits to the data, since the measurements were not made at exactly the same velocities between the runs. For this particular case the percentage difference in pressure gradient decreased as the superficial velocity increased.

Table 2 Calculated pressure reductions for batch one test

\begin{tabular}{llll}
\hline $\begin{array}{l}\text { Velocity } \\
(\mathbf{m} / \mathbf{s})\end{array}$ & $\begin{array}{l}\text { Pressure gradient } \\
\text { control }(\mathbf{k P a} / \mathrm{m})\end{array}$ & $\begin{array}{l}\text { Pressure gradient control } \\
\mathbf{+} \mathbf{0 . 0 5 \%} \text { MAS + 0.015\% salt }(\mathbf{k P a} / \mathbf{m})\end{array}$ & $\begin{array}{l}\text { Difference in pressure } \\
\text { gradient }(\%)\end{array}$ \\
\hline 1.0 & 2.53 & 1.84 & -27 \\
1.5 & 2.60 & 1.99 & -23 \\
2.0 & 2.70 & 2.20 & -19 \\
2.5 & 2.83 & 2.47 & -13 \\
\hline
\end{tabular}

Figure 10 shows the dried behaviour of samples taken during the first batch of tests. The main point of interest is that there were free fine particles 'floating' in the control and the control + $0.05 \%$ MAS samples, which have dried free from the main body of the sample and therefore 'curled'. The control $+0.05 \%$ MAS $+0.015 \%$ salt sample, however, has all of the fine material subsumed into the total mass and therefore dried as a solid mass, with no free fines to curl on top of the sample. This behaviour may be of interest in tailings applications. These observed behaviours are consistent with the rheology tests, where the combination of the MAS and salt was shown to provide an increase in the yield stress of the fines fraction. 


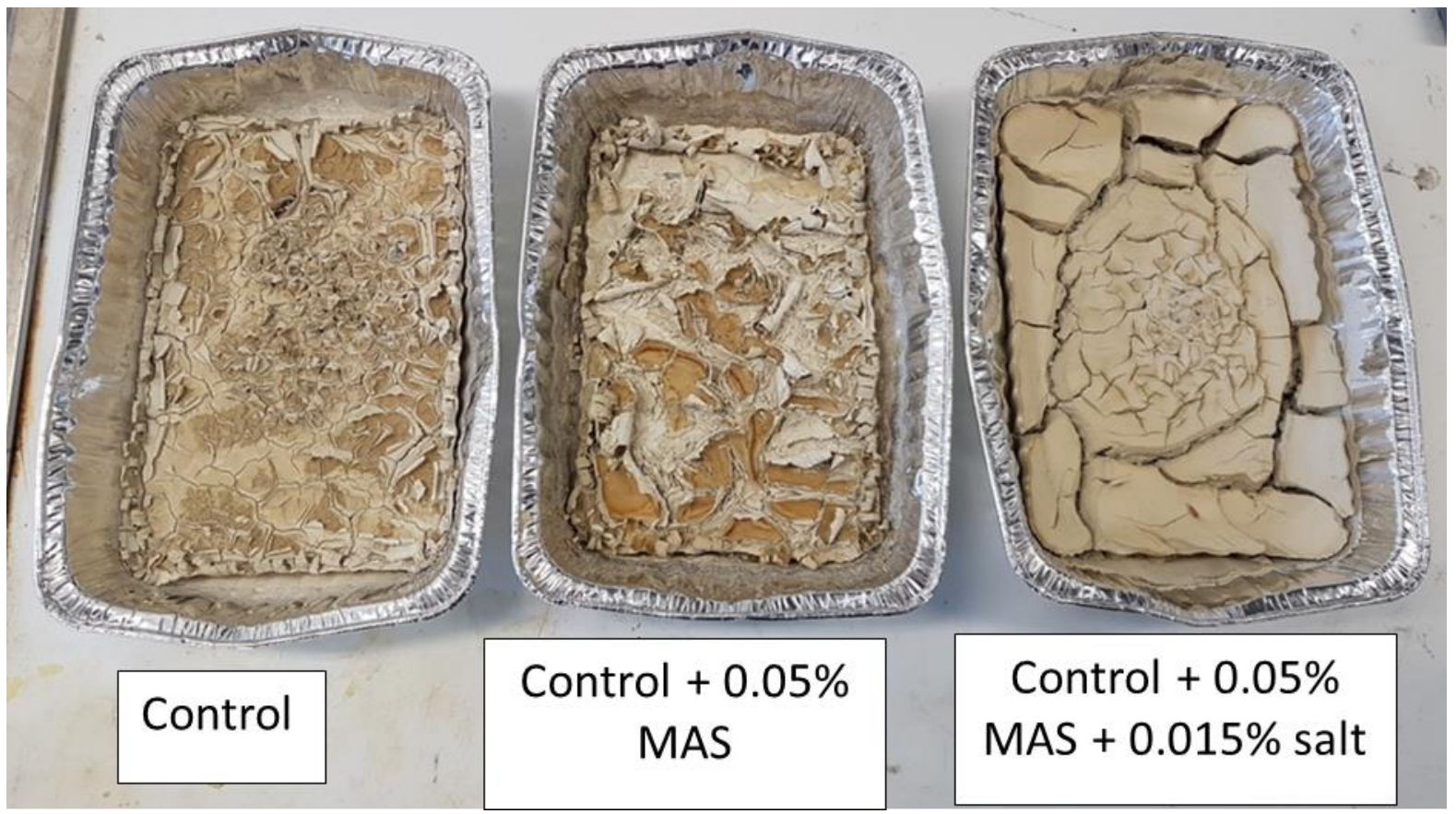

Figure 10 Dried samples from pipe test

The presence of stratified flow indicates that the mechanism of the pressure gradient reduction is not related to fully suspending the coarse particles for the suspensions tested here. However, there are other mechanisms which influence the behaviour of pipe flows containing coarse solids. In particular, it is known from the literature that the bed of solids contributes significantly to the frictional loss in stratified pipe flow, especially for larger pipes. Shook et al. (2002) discuss the basic features for stratified flows in Newtonian liquids and discuss the Coulombic friction which is the contribution to the pressure gradient by coarse particles in contact with the pipe wall in a stratified flow, in this case turbulent. Their model includes a term describing the coefficient of kinematic friction between the pipe wall and the coarse particles in contact with that wall. A fraction of these particles are also suspended by the turbulent action.

Similar models of pipe behaviour have been developed for the case of coarse particles in a more complex suspension which can contain non-Newtonian behaviour. These models are discussed in Pullum et al. (2004), Matousek et al. (2015) and Rojas and Saez (2012). They include components due to the friction of the bed of solids on the pipe wall.

Matousek et al. (2015) express the shear stress (which contribute to the overall pipe pressure gradient) at the pipe wall under the bed of solids as a function of the submerged weight of the bed and a friction coefficient. Both of these can be influenced by the rheology of the carrier fluid, especially if it has a yield stress, as the yield stress can partially support particles if the shear within the bed itself is small, reducing the submerged weight and hence the normal force between the solids and the pipe wall. The yield stress may also influence the packing behaviour of the bed of solids. These aspects of bed behaviour are also discussed in Talmon et al. (2014). The friction coefficient may also be subject to modification due to a lubrication effect.

It is of interest to understand the effect of increasing the rheology of the active part of a suspension for realistic pipe sizes to determine the utility of additives, which can increase rheology with relatively small doses. Some calculations are presented here to illustrate the effect of carrier fluid rheology changes on pipe flow for both homogenous and stratified cases.

The first example considers a $300 \mathrm{~mm}$ diameter pipe with a suspension dominated by fines such that stratification can be neglected. The rheology is assumed to follow the Bingham plastic model. Calculations in the turbulent regime used the Thomas and Wilson (2007) model for the smooth wall case. The calculated flow curves are seen in Figure 11 for a range of rheologies. If the flow regime of interest is laminar, increasing the viscosity leads to an increased pressure gradient in the pipe. However, if the flow regime is turbulent for 
the low yield stresses in this example, as indicated by the arrow, an increase in carrier fluid viscosity leads to a delayed transition to turbulence and consequent reduction in pressure gradient in the pipe for the range of viscosities considered here.

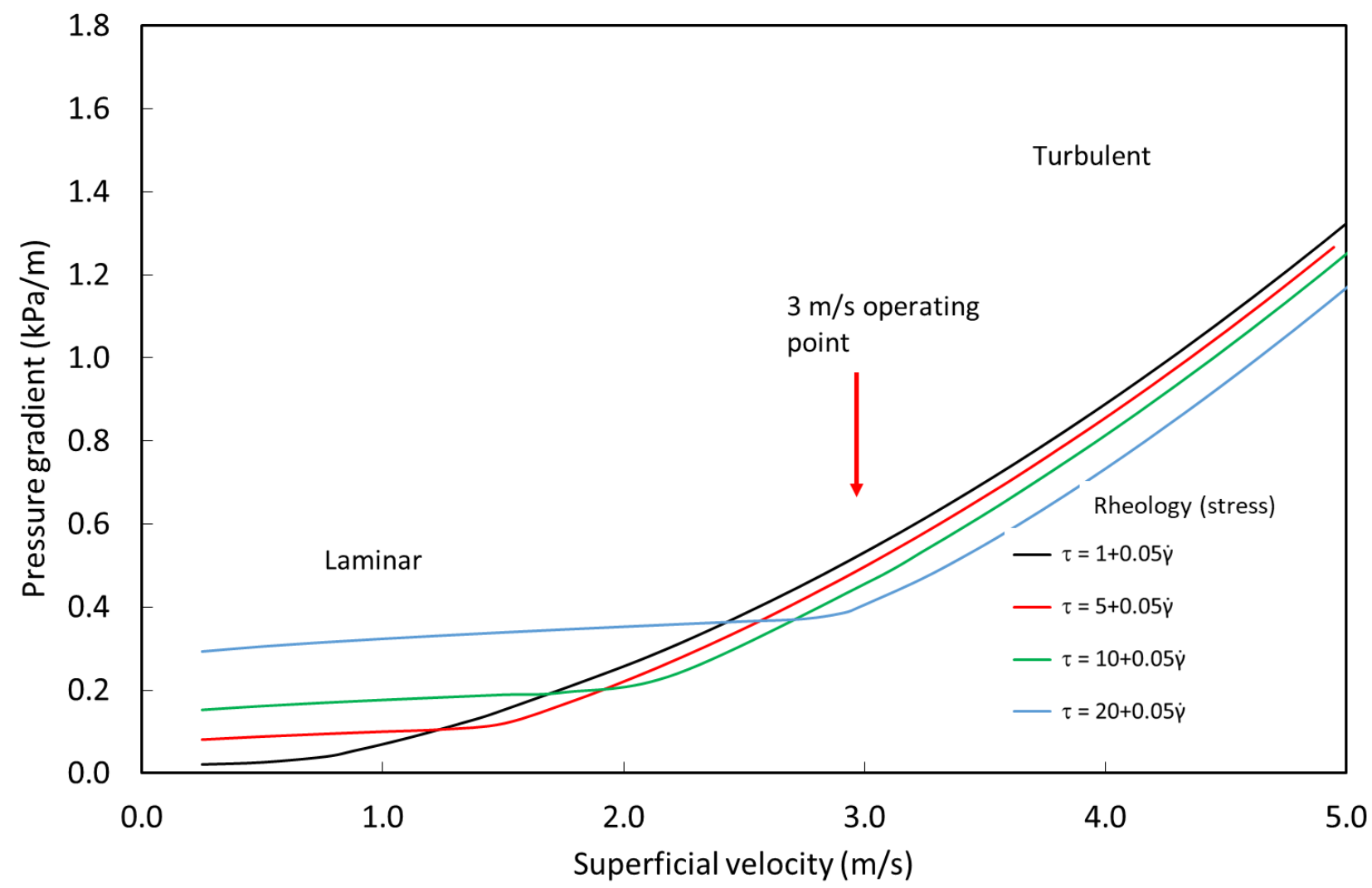

Figure 11 Homogeneous calculations, Bingham plastic model non-Newtonian fluids, $300 \mathrm{~mm}$ pipe

The second example considers the case where a coarse solids fraction is present in the suspension, in this case at $30 \%$ by volume. A more complex model is required to calculate the flow behaviour, and in this case the two-layer approach of Pullum et al. (2004) is used where the suspension is divided into the coarse solids and the carrier fluid. The results are shown in Figure 12. It can be seen that the laminar flow regime is relatively insensitive to the carrier rheology (although it must be stated that there can be an impact here if the friction coefficient is a strong function of the rheology, not considered in the present example). Again, with a typical operating point of $3 \mathrm{~m} \mathrm{~s}^{-1}$, in the turbulent regime, the pressure gradient is reduced for the higher viscosity carrier fluid.

These calculations indicate that increasing the viscosity of the whole suspension (for the fines-dominated case) or the carrier fluid (for a case where there is a significant coarse fraction of inert solids) can reduce the pipe pressure gradient provided that the operating point is within the turbulent regime for low carrier fluid yield stresses.

An increased viscosity can also have a beneficial effect on the minimum conveying velocity, arguably more important than the pressure gradient as discussed by Thomas (2014). An example calculation of minimum conveying velocity has been performed using the method of Gillies et al. (2000). This calculation is for Newtonian fluids only for the case of a $300 \mathrm{~mm}$ pipe and $0.5 \mathrm{~mm}$ particles. The results as a function of viscosity are shown in Figure 13, where the effect of increasing viscosity is to reduce the minimum conveying velocity as would be expected. The delay in the transition to turbulence may also result in less erosion. 


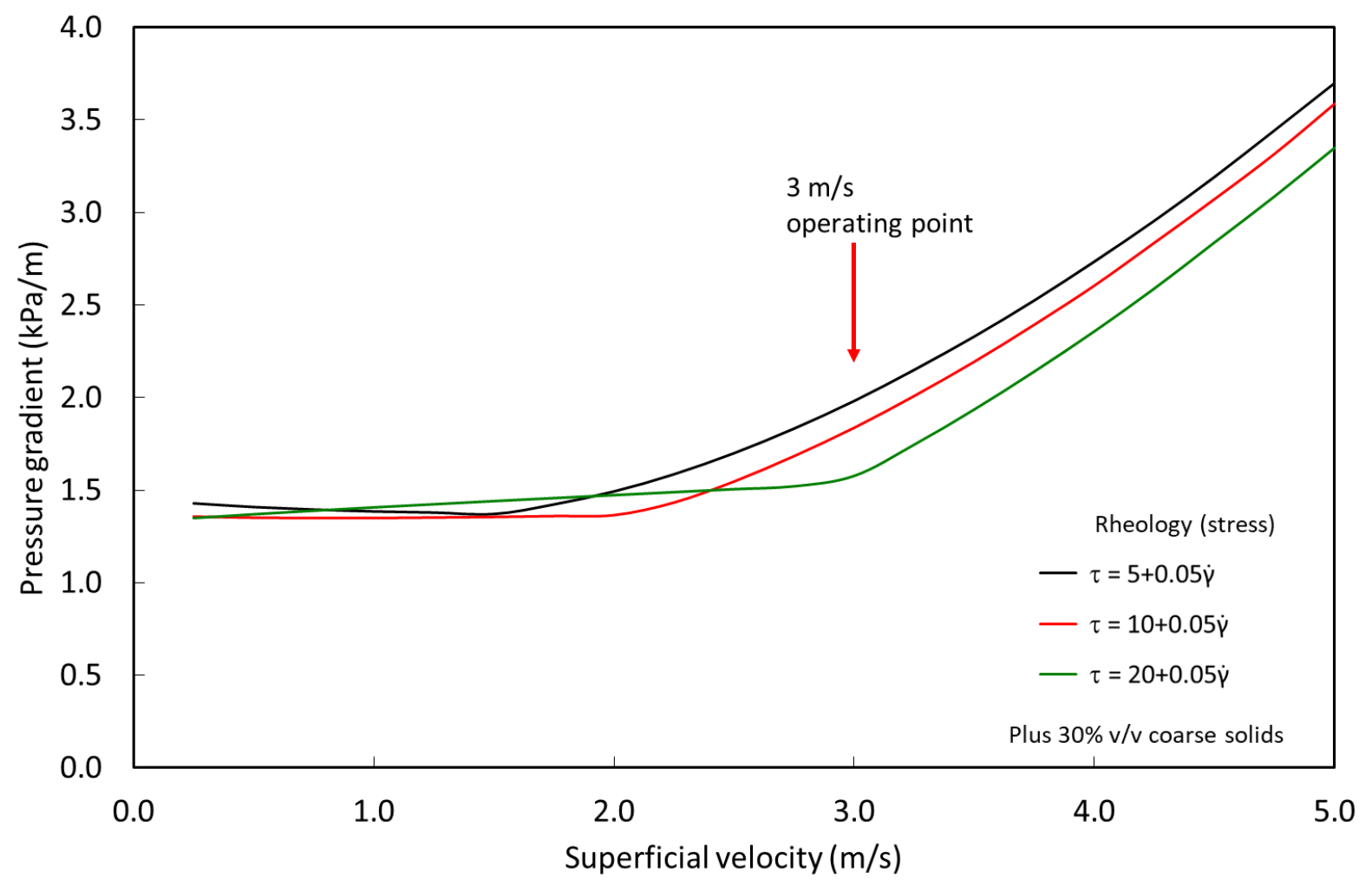

Figure 12 Heterogeneous calculations, $300 \mathrm{~mm}$ pipe, Bingham plastic carrier fluids plus $30 \%$ by volume coarse solids

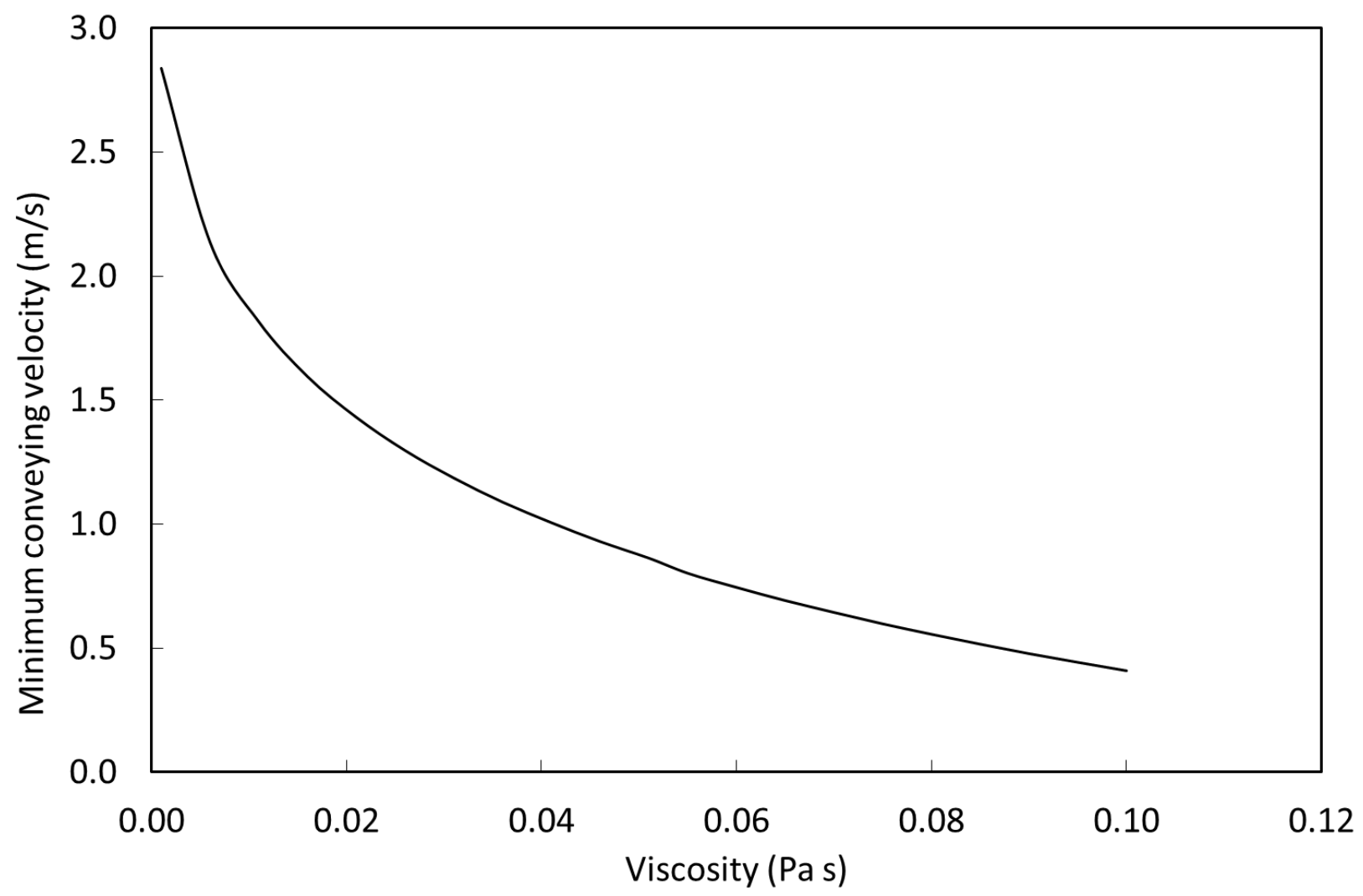

Figure 13 Minimum conveying velocity calculated using the Gillies et al. (2000) method. $300 \mathrm{~mm}$ pipe, $0.5 \mathrm{~mm}$ particles, Newtonian carrier fluid 


\section{Conclusion}

The following conclusions were drawn from the test program:

- Regardless of the additives to the slurry, stratified flow was observed in the pipe for the test results presented here.

- The combination of MAS and salt as additives led to a reduced pressure gradient in the pipe. This combination also had an easier pipe restart from shutdown. The effect is most likely due to the modification of the bed of solids and its frictional behaviour with the changed rheology, particularly in turbulent flows. The minimum conveying velocity was also reduced.

- It was possible to restart without problems after two days for the run where the $0.75 \%$ salt and $0.1 \%$ MAS case was used. This would have not been attempted with the lower viscosity control suspensions due to the risk of blockage.

- Dried samples taken from the pipe tests showed markedly different behaviour for the MAS plus salt case compared with the other samples, with little segregation of the different-size fractions.

- Calculations indicate that increasing the viscosity of homogeneous suspensions or carrier fluids in heterogeneous suspensions can be beneficial in the pipe flow in the turbulent regime.

Future research options could include examining the effect of additives on different clay-based model suspensions, as well as industrial samples.

\section{Acknowledgement}

The authors are grateful to Dean Harris for his work in setting up the pipe rig and for the support of Active Minerals Australia for this work.

\section{References}

Gillies, RG, Schaan, J, Sumner, RJ, Mckibben, MJ \& Shook, CA 2000, 'Deposition velocities for Newtonian slurries in turbulent flow', The Canadian Journal of Chemical Engineering, vol. 78, no. 4, pp. 704-708.

Matousek, V, Penik, V, Pullum, L \& Chryss, A 2015, 'Experimental study of bed friction in stratified flow with viscoplastic carrier in pipe', in J Sobota and C van Rhee (eds), Proceedings of the 17th International Conference on Transport and Sedimentation of Solid Particles, Wroclaw University of Environmental and Life Sciences, Wroclaw, pp. 175-184.

Pullum, L, Graham, LJW \& Slatter, P 2004, 'A non-Newtonian two-layer model and its application to high density hydrotransport', in N Heywood (ed.), Proceedings of the 16th International Conference on Hydraulic Transport of Solids, vol. 2, BHR Group, Cranfield, pp. 579-594.

Rojas, MR \& Saez, AE 2012, 'Two-layer model for horizontal pipe flow of Newtonian and Non-Newtonian settling dense slurries', Industrial \& Engineering Chemistry Research, vol. 51, no. 20, pp. 7095-7103.

Sanders, RS \& Sumner, RJ (eds) 2014, Proceedings of the 19th International Conference on Hydrotransport, BHR Group, Cranfield.

Shook, CA, Gillies, RG \& Sanders, RS 2002, Pipeline Hydrotransport with Applications in the Oil Sands Industry, Saskatchewan Research Council publication number 11508-1E02, Saskatchewan Research Council Pipe Flow Technology Centre, Saskatoon.

Talmon, AM, van Kesteren, WGM, Mastbergen, DR, Pennekamp, JGS \& Sheets, B 2014, 'Calculation methodology for segregation of solids in non-Newtonian carrier fluids', in RJ Jewell, AB Fourie, PS Wells and D van Zyl (eds), Proceedings of the 17th International Seminar on Paste and Thickened Tailings, InfoMine Inc., Vancouver, pp. 139-153.

Thomas, AD 2014, 'Slurries of most interest to the mining industry flow homogeneously and the deposit velocity is the key parameter', Proceedings of the 19th International Conference on Hydrotransport, BHR Group, Cranfield, pp. 239-252.

Thomas, AD \& Wilson, KC 2007, 'Rough-wall and turbulent transition analyses for Bingham plastics', Journal of the South African Institute of Mining and Metallurgy, vol. 107, no. 6, pp. 359-364. 